\title{
COMPARISON BETWEEN ARTIFICIAL BEE COLONY AlgorithM, SHUFFLEd FROG LEAPING ALGORITHM AND NERO-FUZZY SYSTEM IN DESIGN OF OPTIMAL PID CONTROLLERS
}

\author{
Fatemeh Masoudnia $^{1}$ and Somayyeh Nalan Ahmadabad ${ }^{2}$ and Maryam \\ Kouzehgar $^{3}$ \\ ${ }^{1,2}$ Department of Electrical Engineering, Sofyan Branch, \\ Islamic Azad University, Sofyan, Iran \\ ${ }^{1}$ f_masoudnia@yahoo.com \\ ${ }^{2}$ nalansomayyeh@gmail. com \\ ${ }^{2}$ Control Engineering Department Faculty of Electrical \& Computer \\ Engineering Tabriz, Iran \\ maryamkouzehgaregmail.com
}

\begin{abstract}
In this paper three optimum approaches to design PID controller for a Gryphon Robot are presented. The three applied approaches are Artificial Bee Colony, Shuffled Frog Leaping algorithms and nero-fuzzy system. The design goal is to minimize the integral absolute error and reduce transient response by minimizing overshoot, settling time and rise time of step response. An Objective function of these indexes is defined and minimized applying Shuffled Frog Leaping (SFL) algorithm, Artificial Bee Colony (ABC) algorithm and Nero-Fuzzy System $(F N N)$. After optimization of the objective function, the optimal parameters for the PID controller are adjusted. Simulation results show that FNN has a remarkable effect on decreasing the amount of settling time and rise-time and eliminating of steady-state error while the SFL algorithm performs better on steady-state error and the ABC algorithm is better on decreasing of overshoot. In steady state manner, all of the methods react robustly to the disturbance, but FNN shows more stability in transient response.
\end{abstract}

\section{KEYWORDS}

Robotics, PID controller, Gryphon, dynamic equations, shuffled frog leaping, artificial bee colony, nero-fuzzy system 


\section{INTRODUCTION}

PID controller (proportional-integral-derivative controller) benefiting from major advantages such as simple structure and wide-domain performance, is one of the most frequently used controllers. There are three parameters, on the basis of which, the PID controller is defined, i.e. proportional gain $K_{\mathrm{p}}$, integral gain $K_{\mathrm{i}}$, and derivative gain $K_{\mathrm{d}}$. The traditional procedure to assign these parameters is a sophisticated and time-consuming try and error process. Thus several new methods are recommended to reduce the complexity of tuning PIDs. These methods, having the biological or social inspiration as a common theme, are accomplished within evolutionary algorithms such as Binary and Continuous Genetic algorithms [1], Ant Colony Optimization [2], Continuous and Discrete Particle Swarm Optimization [3],[4] and different types of Honey Bee Colony Algorithms [5],[6].

In this approach, working on Gryphon robot which includes 5 joints, we must design five PID controllers; each for one joint. Thus we must first verify the dynamic equations for Gryphon robot. Then we will formulate the PID controller designation problem as an optimization problem with an objective function which is supposed to be minimized by adjusting the four performance indexes, i.e. the maximum overshoot, the rise time, the settling time and the integral absolute error of step response. And finally we will minimize the defined objective function for each joint by applying Shuffled Frog Leaping (SFL) algorithm, Artificial Bee Colony (ABC) algorithm and Nero-Fuzzy System (FNN).

The paper is organized in 6 sections to illustrate the case. Section 2 includes a brief description of the system under-investigate and presents the verification of the dynamic equations of the robot. Section 3 reviews the utilized intelligent algorithms and FNN and in section 4 the problem formulation is explained. Section 5 presents the simulation results and analyses them and section 6 concludes the paper.

\section{A BRIEF DESCRIPTION OF THE SYSTEM}

As shown in Figure 1, Gryphon is a robot with 5 revolute joints. Thus this robot benefits from 5 degrees of freedom. The first three joints which are called shoulder elbow and wrist respectively, are supposed to determine the position of the end-effecter while the last two joints are responsible for the orientation of the end-effecter. Fast and smooth movement while maintaining the high precision is one of the major characteristics of this robot. This robot is controlled by four microprocessors, one of which is to determine the position of the axis, and two are motor controllers and the last one is supposed to support the others and communicate with the host computer. Each joint is moved by a stepper motor while there is feedback on the corresponding encoder to realize the closed-loop control. In this robot the utilized gear ratio is high enough to assume all joints independent from one another.

We obtained the dynamic equations of all five joints. Table 1 shows transfer functions of all five joints that are obtained from the so-called dynamic equations, adding the process of obtaining which sounds to need a vast space for complicated equations and that is why we have avoided them here. In the transfer functions in Table 1, $\alpha$ stands for the amount of disturbance exerted on the system. For an undisturbed system $\alpha$ is set to zero. 


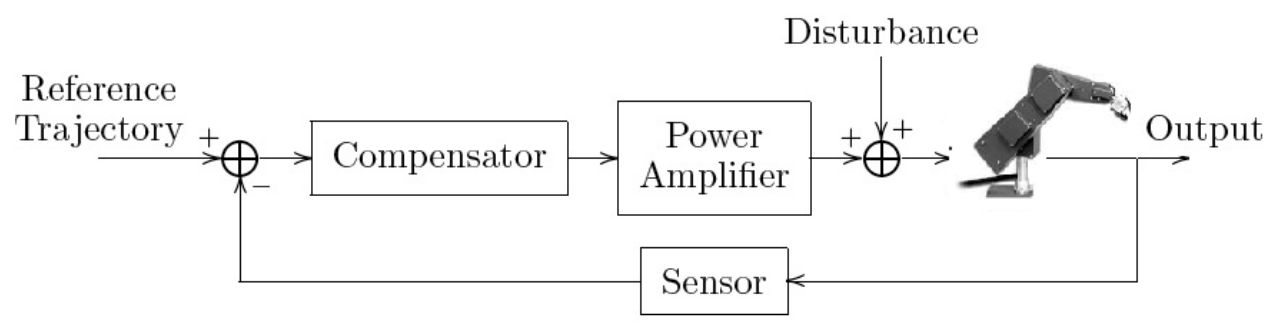

Figure 1. Joint model for Gryphon

\section{THE UTILIZED INTELLIGENT ALGORITHMS}

\subsection{The Shuffled Frog Leaping Algorithm (SFL)}

In the SFL, the population consists of a set of frogs (solutions) that is partitioned into subsets referred to as memeplexes. The different memeplexes are considered as different cultures of frogs, each performing a local search. Within each memeplex, the individual frogs hold ideas, that can be influenced by the ideas of other frogs, and evolve through a process of memetic evolution. After a defined number of memetic evolution steps, ideas are passed among memeplexes in a shuffling process [7]. The local search and the shuffling processes continue until defined convergence criteria are satisfied [8].

Table 1. Transfer Functions of Gryphon Joints

\begin{tabular}{|c|c|}
\hline Joint Number & Transfer Function \\
\hline Joint 1 & $\frac{-.0005 s+.1012}{.0448 s^{2}+.5581 s-.0126+\alpha}$ \\
\hline Joint 2 & $\frac{-.0031 s+.1012}{.0221 s^{2}+.6761 s-.0125+\alpha}$ \\
\hline Joint 3 & $\frac{.0005 s+.1013}{.0210 s^{2}+.3968 s-.0130+\alpha}$ \\
\hline Joint 4 & $\frac{-.0022 s+.1005}{.0096 s^{2}+1.4940 s-.0051+\alpha}$ \\
\hline Joint 5 & $\frac{.0276 s+.1005}{.0539 s^{2}+1.4210 s-.0059+\alpha}$ \\
\hline
\end{tabular}

An initial population of $\mathrm{P}$ frogs is created randomly. For S-dimensional problems ( $\mathrm{S}$ variables), a frog $\mathrm{i}$ is represented as $\mathrm{X}_{\mathrm{i}}=\left(\mathrm{X}_{\mathrm{i} 1}, \mathrm{X}_{\mathrm{i} 2}, \ldots \ldots . ., \mathrm{X}_{\mathrm{is}}\right)$. Afterwards, the frogs are sorted in a scending order according to their fitness. Then, the entire population is divided into $\mathrm{m}$ memeplexes, each containing $\mathrm{n}$ frogs (i.e. $\mathrm{P}=\mathrm{m} \times \mathrm{n}$ ). In this process, the first frog goes to the first memeplex, the second frog goes to the second memeplex, frog $(m)$ goes to the mthmemeplex, and frog $(m+1)$ goes back to the first memeplex, etc.

Within each memeplex, the frogs with the best and the worst fitnesses are identified as $X_{b}$ and $\mathrm{X}_{\mathrm{w}}$, respectively. Also, the frog with the global best fitness is identified as $\mathrm{X}_{\mathrm{g}}$. Then, a process is applied to improve only the frog with the worst fitness (not all frogs) in each cycle. Accordingly, the position of the frog with the worst fitness is adjusted as follows: 


$$
\begin{aligned}
& \text { Change in frog position }\left(D_{i}\right)=\operatorname{rand} \times\left(X_{b}-X_{w}\right) \\
& \text { New position } X_{w}=\text { current position } X_{w}+D_{i} ; \\
& \quad D_{\max } \geq D_{i} \geq-D_{\max }
\end{aligned}
$$

Where rand is a random number between 0 and 1; and $\mathrm{D}_{\max }$ is the maximum allowed change in a frog's position.

If this process produces a better solution, it replaces the worst frog. Otherwise, the calculations in Eqs (1) and (2) are repeated but with respect to the global best frog (i.e. $X_{\mathrm{g}}$ replaces $\mathrm{X}_{\mathrm{b}}$ ). If no improvement becomes possible in this case, then a new solution is randomly generated to replace that frog. The calculations then continue for a specific number of iterations [8]. Accordingly, the main parameters of SFL are: number of frogs P; number of memeplexes; number of generation for each memeplex before shuffling; number of shuffling iterations; and maximum step size.

\subsection{The Artificial Bee Colony Algorithm (ABC)}

Karaboga analyzes the foraging behavior of honey bee swarm and proposes a new algorithm simulating this behavior for solving multi-dimensional and multi-modal optimization problems, called Artificial Bee Colony (ABC) [5]. The main steps of the algorithm are:

1) send the employed bees onto the food sources and determine their nectar amounts;

2) calculate the probability value of the sources with which they are preferred by the onlooker bees;

3) stop the exploitation process of the sources abandoned by the bees;

4) send the scouts into the search area for discovering new food sources, randomly;

5) memorize the best food source found so far.

In the algorithm, an artificial bee colony consists of three groups of bees: employed bees, onlookers and scouts. Employed bees are associated with a particular food source which they are currently exploiting. They carry the information about this particular source and share this information with a certain probability by waggle dance. Unemployed bees seek a food source to exploit. There are two types of unemployed bees: scouts and onlookers. Scouts search the environment for new food sources without any guidance. Occasionally, the scouts can accidentally discover rich, entirely unknown food sources. On the other hand onlookers observe the waggle dance and so are placed on the food sources by using a probability based selection process. As the nectar amount of a food source increases, the probability value with which the food source is preferred by onlookers increases, too. In the ABC algorithm the first half of the colony consists of the employed bees and the second half includes the onlookers. For every food source, there is only one employed bee. Another issue that is considered in the algorithm is that the employed bee whose food source has been exhausted by the bees becomes a scout. In other words, if a solution representing a food source is not improved by a predetermined number of trials, then the food source is abandoned by its employed bee and the employed bee is converted to a scout. 


\subsection{The Nero-Fuzzy System (FNN)}

Neural Networks, using a system based on human brain structure, have the ability to learn how to face new challenges. These types of systems are able to adapt themselves to learn how to become compatible with new conditions which have not formerly experienced. Fuzzy logic, based on rules that includes a knowledgebase, is formed fuzzy rules of "if - then". These rules are made of simple and understandable words. The experts who know the system behavior define these rules by means of natural language. Through combination of Neural Networks and Fuzzy logic NeroFuzzy systems are accessible. Any Nero-Fuzzy system is a Neural Network which learns how to classify data using Fuzzy rules and Fuzzy sets.

A Nero-Fuzzy network is a network with feedback comprising five layers. These layers are:

1) Input layer;

2) Input membership functions of fuzzy;

3) Fuzzy rules;

4) Output membership functions of fuzzy;

5) Output layer;

Figure 2 shows a general view of this network. In this figure the network has two deterministic inputs, $\mathrm{T}$ and $\mathrm{H}$. And it produces a deterministic output, Q. In a system, number of inputs and outputs are changeable according to our requirement. In this paper there are four inputs and three outputs.

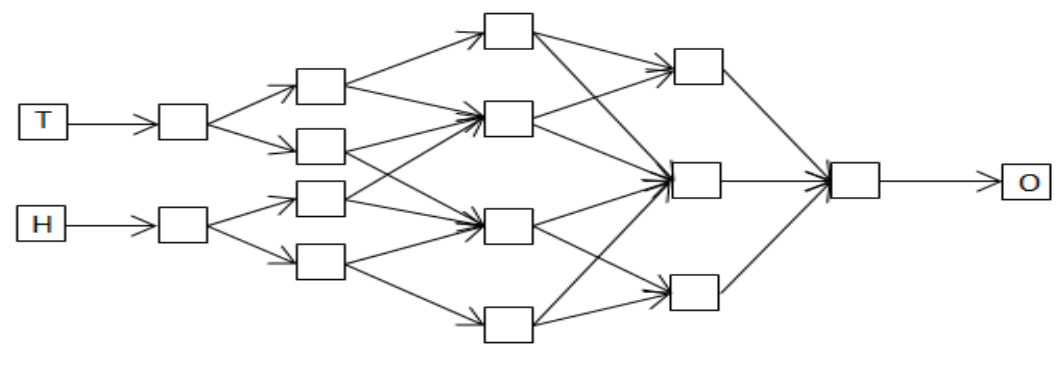

Layer 1 Layer 2 Layer $3 \quad$ Layer $4 \quad$ Layer 5

Figure 2. A general view of a nero-fuzzy network with five layers.

Work of first layer is only transmission of deterministic inputs to the second layer of network. In the second layer, deterministic inputs become fuzzy. In this paper, we used Triangular membership functions for fuzzyfication. Third layer includes set of fuzzy rules. In this layer, each neuron is a fuzzy rule. System can be set with some initial Fuzzy rules and the network adjusts the weights in a way which gives the best response. In some cases it is possible to start the system without initial rules. In such cases system learns the rules by itself and adjusts the weights based on them.

Forth layer in the network includes the neurons presenting the membership functions for different and possible outputs of Fuzzy rules. Fifth and the last layer combines and defuzzy tune different outputs. 
FNN method simulate by ANFIS, train data for ANFIS obtain from several hand tuning of PID parameters. Train data upload by ANFIS and use for FNN learning. After learning, by uploading of ANFIS to simulation environment it will set the PID parameters.

\section{PROBLEM ForMULATION TO DESIGN PID CONTROLLERS}

The transfer function of the PID controller is considered as follows.

$$
G_{c}(s)=K_{p}+\frac{K_{i}}{s}+K_{d} s
$$

Where $\mathrm{K}_{\mathrm{p}}, \mathrm{K}_{\mathrm{i}}$ and $\mathrm{K}_{\mathrm{d}}$ are proportional, integral and derivative gains respectively. In the time domain the characteristics of step response such as rise time $t_{r}$, overshoot $M_{p}$, settling time $t_{s}$ and steady state error $\mathrm{E}_{\mathrm{ss}}$ are considered as a performance criterion for PID controller. We use a function of these indexes as the objective function which is illustrated by Equation (4).[9]

$$
f(k)=\left(1-e^{-\beta}\right)\left(M_{p}+E_{s s}\right)+e^{-\beta}\left(t_{s}-t_{r}\right)
$$

Where $\mathrm{K}$ is a vector whose elements are the PID controller gains. With which the step response of the system is simulated and the values of Mp, Ess, ts and tr are obtained to evaluate $\mathrm{f}(\mathrm{k})$.

$$
K=\left[K_{d} K_{p} K_{i}\right]
$$

And $\beta$ is a weighting factor whose value effects the four indexes. This value, if set to be smaller than 0.7, results in reduction of $t_{\mathrm{r}}$ and $\mathrm{t}_{\mathrm{s}}$. Otherwise, if set to values larger than 0.7 , end in a decrease in $E_{s s}$ and $M_{p}$ while setting $\beta=0.7$ will cause the four parameters to effect similarly on objective function.

On both of the compared algorithms, the vector $\mathrm{K}$ is defined as a member of population. The algorithms start from a random population. During each iteration of the algorithm, the elements of each member of population are substituted in the controller transfer function $\mathrm{G}_{\mathrm{c}}(\mathrm{s})$ and the step response is calculated for each member of the population, on the basis of which, $t_{r}, t_{s}, E_{s s}$ and $M_{p}$ are calculated and substituted in the defined objective function. The goal of all the algorithms is to minimize the objective function through the strategies illustrated above.

\section{SIMULATION RESULTS}

\subsection{Parameter Evaluation on the Algorithms}

On both of the algorithms, all of the controller parameters, are restricted to the interval $[0,30]$. For both of the algorithms, 30 runs, with 5000 function evaluations included in each run, have been carried out to have the average answer which is more reliable. To determine the robustness of the system, all simulations have been run under the effect of a disturbance factor. The parameter evaluation for each utilized algorithm is as follows. 


\subsubsection{SFL Algorithm}

Population size $=50$, The number of memeplexes $=10$, Memetic evolution steps before shuffling=10, Maximum number of function evaluation=5000.

\subsubsection{ABC Algorithm}

Population size $=20$, The number of employee bees $=0.3$ population size, The number of on-looker bees $=0.3$ population size, The number of scout bees $=0.4$ population size, Maximum number of function evaluation $=5000$.

\subsection{Parameter Evaluation on FNN}

FNN generate by grid partition with 3 member ship functions and train by hybrid method. Number of MFs=3, Input MF type=gbellmf, Output MF type=constant.

\subsection{The results}

Tables 2 and 3 respectively present the simulation results of SFL and ABC algorithm for all of the five joints with $\beta=0.7$. Table 4 indicates the same values for FNN. The step responses for each of the five motors are shown on figures 3 to 17. In these pictures, the graph shown with direct line indicates the response of the undisturbed system while the other two show the disturbed systems with different disturbances $(\alpha=0.1$ and $\alpha=-0.1)$.

Applying the $\mathrm{kp}$, ki, kd values obtained from the intelligent algorithms, simulation results show that FNN has a remarkable effect on decreasing the amount of settling time and rise-time and eliminating of steady-state error because considering the average value of the 5 joints according to Tables 2 to 4 , it is obviously seen in Table 5 that the average settling-time, the average rise-time and the average steady-state error is far less in FNN in comparison to ABC and SFL. while the SFL algorithm compared to ABC performs slightly better on steady-state error ( 0.0006 vs. 0.0009 ) and the $\mathrm{ABC}$ algorithm is better on decreasing of overshoot. In steady state manner all of the methods react robustly to the disturbance, but FNN shows more stability in transient response.

Table 2. Simulation Results applying SFL algorithm

\begin{tabular}{|c|c|c|c|c|c|c|c|}
\hline \multirow{2}{*}{ Joints } & \multicolumn{4}{|l|}{ PID Parameters } & \multicolumn{4}{l|}{ Step Response Parameters } \\
\cline { 2 - 8 } & $\boldsymbol{K}_{\boldsymbol{d}}$ & $\boldsymbol{K}_{\boldsymbol{v}}$ & $\boldsymbol{K}_{\boldsymbol{I}}$ & $\boldsymbol{M}_{\boldsymbol{v}}$ & $\boldsymbol{E}_{\boldsymbol{z}}$ & $\boldsymbol{t}_{\boldsymbol{r}}$ & $\boldsymbol{t}_{\boldsymbol{s}}$ \\
\hline Joint 1 & 9.8832 & 23.4645 & 6.0537 & .0417 & .0000 & 1.3371 & 6.7864 \\
\hline Joint 2 & 2.0628 & 26.3914 & 14.1972 & .0861 & .0000 & .5354 & 4.0012 \\
\hline Joint 3 & 12.2736 & 24.8578 & 3.3813 & .0253 & .0001 & 1.7084 & 7.8000 \\
\hline Joint 4 & 2.2865 & 22.9050 & 14.4540 & .1038 & .0000 & .5998 & 3.8516 \\
\hline Joint 5 & 1.8861 & 21.5737 & .6692 & .0130 & .0029 & 1.1362 & 5.3366 \\
\hline
\end{tabular}


Table 3. Simulation Results applying ABC algorithm

\begin{tabular}{|l|c|c|c|c|c|c|c|}
\hline \multirow{2}{*}{ Joints } & \multicolumn{3}{|l|}{ PID Parameters } & \multicolumn{4}{|c|}{ Step Response Parameters } \\
\cline { 2 - 8 } & $K_{d}$ & $K_{\boldsymbol{s}}$ & $\boldsymbol{K}_{\boldsymbol{I}}$ & $\boldsymbol{M}_{\boldsymbol{v}}$ & $\boldsymbol{E}_{\boldsymbol{z}}$ & $\boldsymbol{t}_{\boldsymbol{r}}$ & $\boldsymbol{t}_{\boldsymbol{s}}$ \\
\hline Joint 1 & 3.1838 & 22.8034 & 1.2721 & .0172 & .0012 & 1.1903 & 5.2351 \\
\hline Joint 2 & 5.5132 & 29.9019 & 3.0487 & .0196 & .0001 & 1.0494 & 5.0728 \\
\hline Joint 3 & .8966 & 20.3226 & 1.1469 & .0201 & .0013 & .9203 & 7.1673 \\
\hline Joint 4 & 1.4114 & 27.2506 & 1.0234 & .0118 & .0019 & .7417 & 2.7938 \\
\hline Joint 5 & 11.7983 & 24.4511 & 3.9375 & .0287 & .0000 & 1.6203 & 7.7243 \\
\hline
\end{tabular}

Table 4. Simulation Results applying FNN algorithm

\begin{tabular}{|l|c|c|c|c|c|c|c|}
\hline \multirow{2}{*}{ Joints } & \multicolumn{4}{|l|}{ PID Parameters } & \multicolumn{4}{l|}{ Step Response Parameters } \\
\cline { 2 - 8 } & $\boldsymbol{K}_{\boldsymbol{d}}$ & $\boldsymbol{K}_{\boldsymbol{v}}$ & $\boldsymbol{K}_{\boldsymbol{I}}$ & $\boldsymbol{M}_{\boldsymbol{v}}$ & $\boldsymbol{E}_{\boldsymbol{z}}$ & $\boldsymbol{t}_{\boldsymbol{r}}$ & $\boldsymbol{t}_{\boldsymbol{s}}$ \\
\hline Joint 1 & 2.1666 & 52.5094 & 8.2394 & .0421 & .0001 & .2822 & 1.7952 \\
\hline Joint 2 & 2.1665 & 52.5157 & 8.2425 & .0422 & .0001 & .2822 & 1.7954 \\
\hline Joint 3 & 2.5001 & 52.5080 & 12.7716 & .0421 & .0000 & .2876 & 2.8411 \\
\hline Joint 4 & 1.9811 & 52.5017 & 15.1483 & .0416 & .0000 & .2665 & 2.9366 \\
\hline Joint 5 & 1.3549 & 38.9996 & 19.6747 & .0839 & .0000 & .3273 & 3.3340 \\
\hline
\end{tabular}

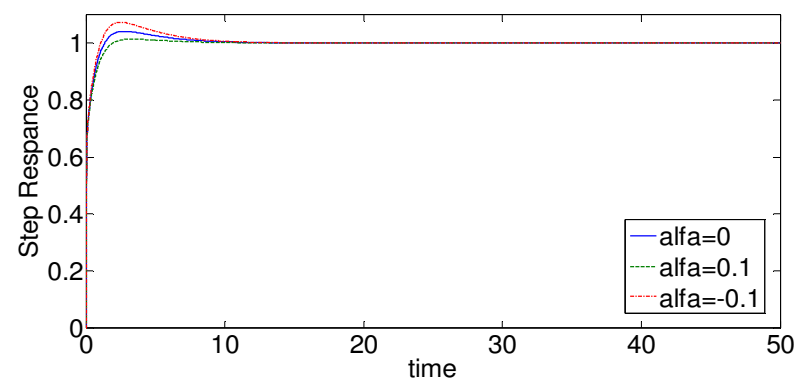

Fig 3. Step responses with SFL applied on motor 1

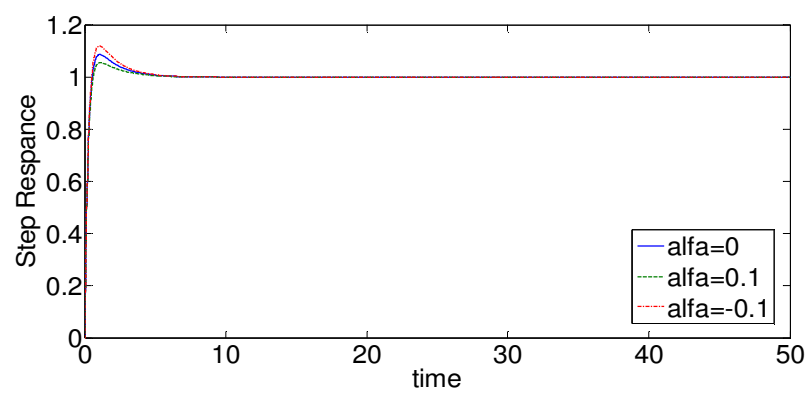

Fig 4. Step responses with SFL applied on motor 2

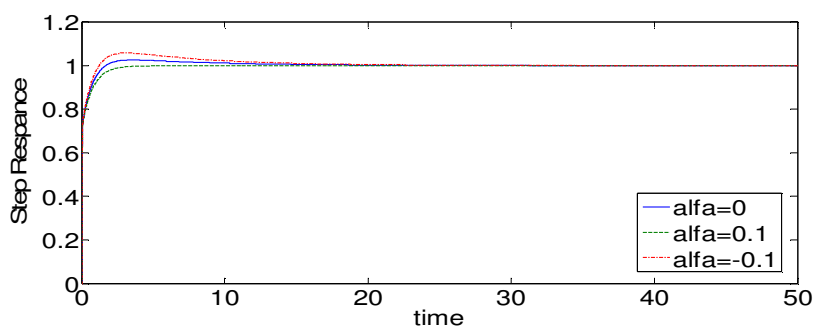

Fig 5. Step responses with SFL applied on motor 3 


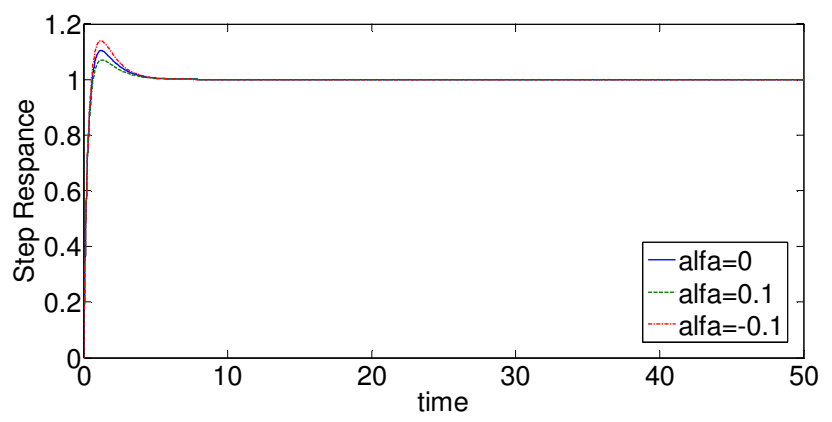

Fig 6. Step responses with SFL applied on motor 4

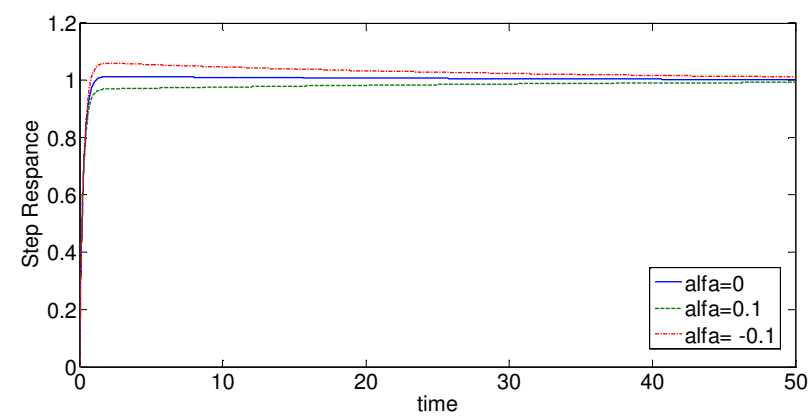

Fig 7. Step responses with SFL applied on motor 5

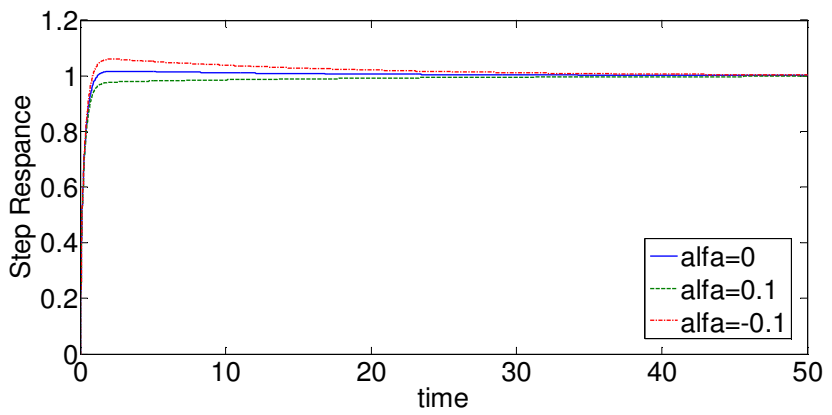

Fig 8. Step responses with ABS applied on motor 1

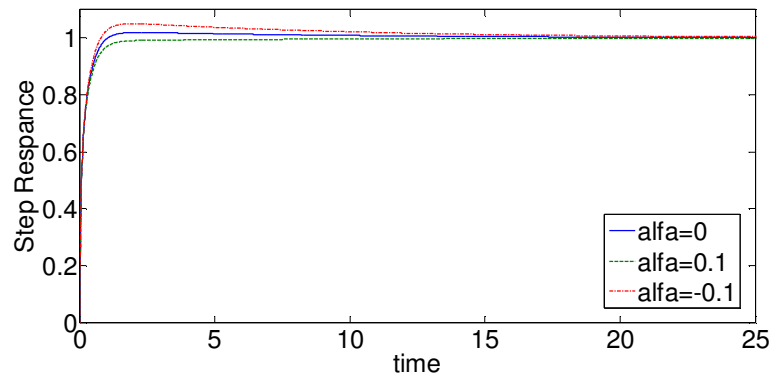

Fig 9. Step responses with ABS applied on motor 2 


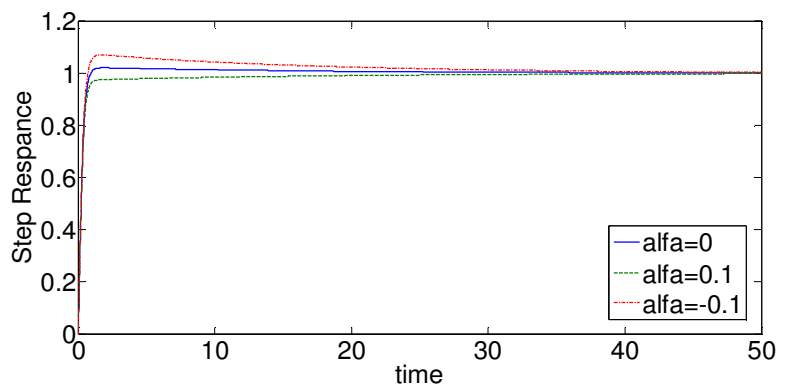

Fig 10. Step responses with ABS applied on motor 3

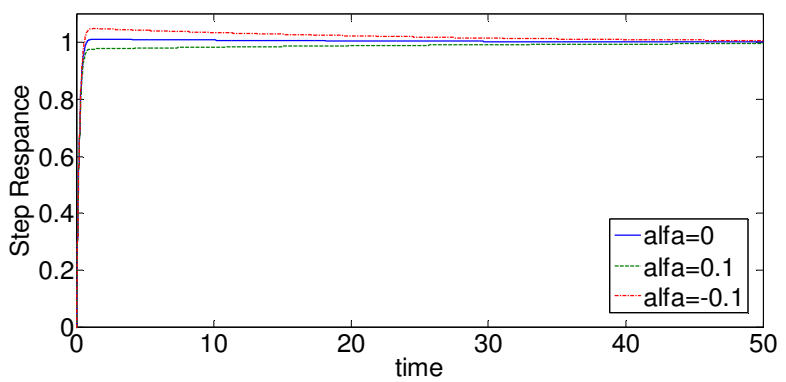

Fig 11. Step responses with ABS applied on motor 4

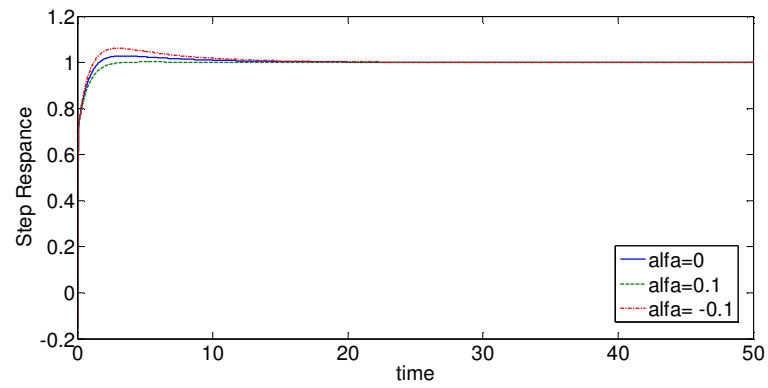

Fig 12. Step responses with ABS applied on motor 5

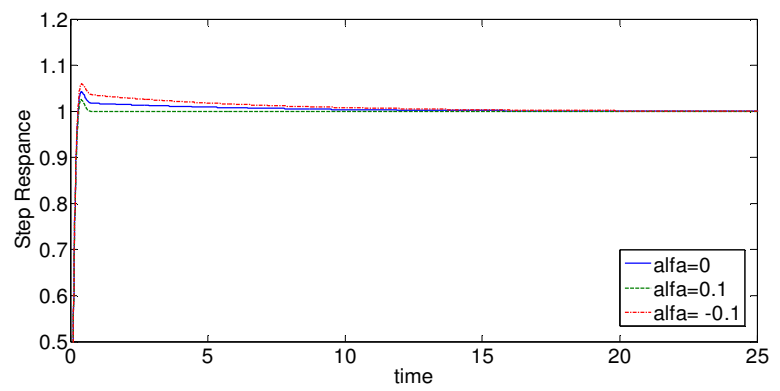

Fig 13. Step responses with FNN applied on motor 1 


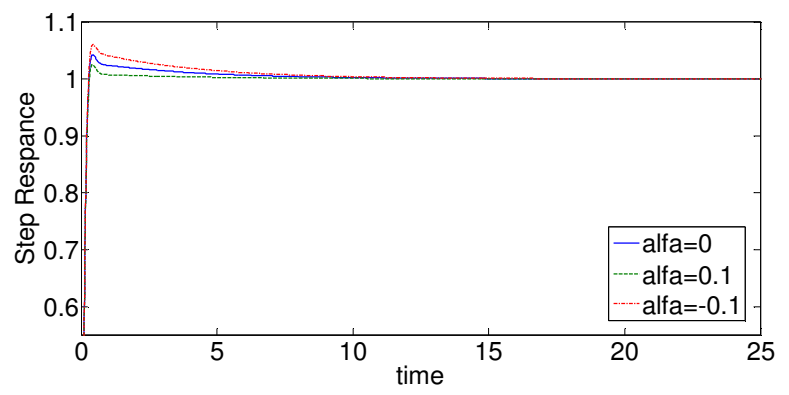

Fig 14. Step responses with FNN applied on motor 2

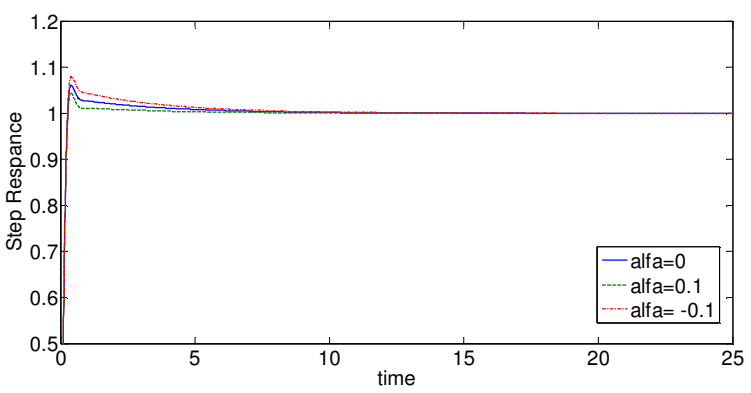

Fig 15. Step responses with FNN applied on motor 3

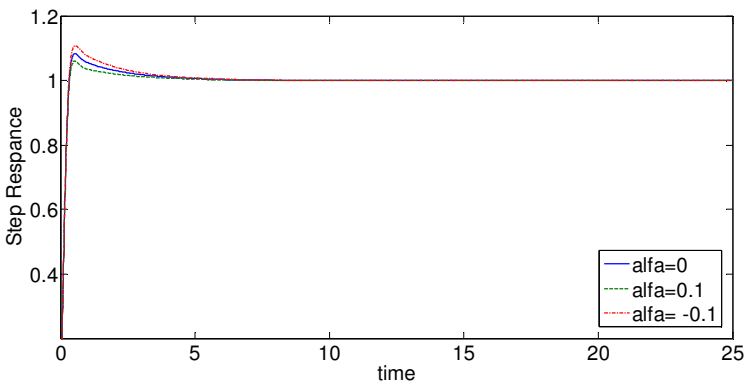

Fig 16. Step responses with FNN applied on motor 4

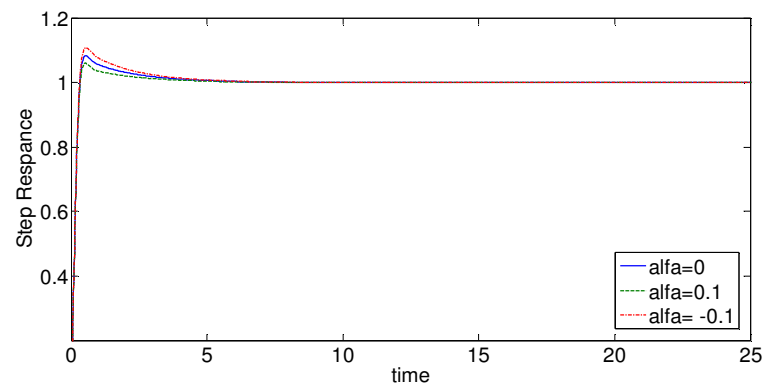

Fig 17. Step responses with FNN applied on motor 5 


\section{CONCLUSION, DISCUSSION AND FUTURE WORKS}

This research begins by introducing the Grypon robot as case-study. Then three methods were applied to design an intelligent PID controller which, unfortunately, is not fast enough to be utilized in on-line applications. The results of applied simulations are reported in the included tables and figures.

Comparing the contents of tables and figures leads to introducing the FNN strong enough in elimination of the steady state error and reduction of the settling time and rise time. On the other hand the ABC algorithm acts better on decreasing the overshoot. Also the act of SFL algorithm is good in elimination of the steady state error.

From the robustness point of view, while applying the disturbance factor, the SFL algorithm and FNN perform stronger in the transient part, however all of the methods detect the main graph in the steady-state manner.

Designing PID controllers with other intelligent algorithms and changing the present controller to an on-line one may be our future field study.

\section{REFERENCES}

[1] R.L. Haupt, S.E. Haupt, Practical Genetic Algorithms, John Wiley, 2004.

[2] M. Dorigo and T. Stützle, “Ant Colony Optimization”, MIT Press, Cambridge, 2004.

[3] R.C. Eberhart and Y. Shi, "Particle Swarm Optimization: Developments, Applications and Resources", IEEE Trans, 2001.

[4] J. Kennedy, and R.C. Eberhart, "A Discrete Binary Version ofthe Particle Swarm Algorithm”, IEEE International Conference on Systems, Man and Cybernetics, 'Computational Cybernetics and Simulation', vol.5,pp.4104-4108, 1997 .

[5] D. Karaboga, B. Basturk, A powerful and efficient algorithm for numerical function optimization:artificial bee colony, Journal of Global Optimization, Volume 39, Issue 3, PP. 459 471, 2007

[6] D. Karoboga, B. Basturk, On the Performance of Artificial Bee Colony (ABC) Algorithm, Applied Soft Computing, pp:687- 697, Vol. 8, Issue 1, 2008.

[7] Liong S-Y, Atiquzzaman Md. Optimal design of water distribution network using shuffled complex evolution. J Inst Eng, Singapore 2004;44(1):93-107.

[8] Eusuff MM, Lansey KE. Optimization of water distribution network design using the shuffled frog leaping algorithm J Water Resour Plan Manage 2003;129(3):210-25.

[9] L. Gaing, A Paticle Swarm Optimization Approch For Optimum Design of PID Controller in AVR System, IEEE International Conference on Energy conversion, pp. 384-391, Vol.19, Issue2, 2004.

[10] E. Elbeltagi, T. Hegazy, and D. Grierson, "Comparison among Five Evolutionary-Based Optimization Algorithms," Advanced Engineering Informatics, vol. 19, no. 1, pp. 43-53, 2005.

[11] Mark W. Spong, Seth Hutchinson, M. Vidyasagar, "Robot Modeling and Control". ISBN: 978-0-47164990-8.

[12] I. Hassanzadeh, H. Jabbari, "Open Architecture of Gryphon Robot for Visual Servo and Teleoperation Tasks", Proceeding of the 4th t International Symposium on Mechatronics and its Applications (ISMA07), Sharjah, U.A.E. March 26-29, 2007 